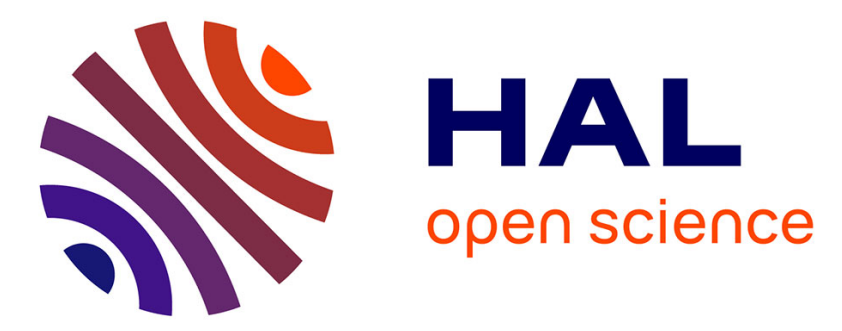

\title{
Engraissement des vaches de réforme de race Limousine
}

C. Malterre, J. Robelin, Jacques Agabriel, P. Bordes

\section{- To cite this version:}

C. Malterre, J. Robelin, Jacques Agabriel, P. Bordes. Engraissement des vaches de réforme de race Limousine. Productions Animales, 1989, 2 (5), pp.325-334. hal-00895880

\section{HAL Id: hal-00895880 \\ https://hal.science/hal-00895880}

Submitted on 1 Jan 1989

HAL is a multi-disciplinary open access archive for the deposit and dissemination of scientific research documents, whether they are published or not. The documents may come from teaching and research institutions in France or abroad, or from public or private research centers.
L'archive ouverte pluridisciplinaire HAL, est destinée au dépôt et à la diffusion de documents scientifiques de niveau recherche, publiés ou non, émanant des établissements d'enseignement et de recherche français ou étrangers, des laboratoires publics ou privés. 
INRA Prod. Anim., $1989,2(5), 325-334$

\section{MALTERRE, J. ROBELIN J. AGABRIEL, P. BORDES*}

INRA Station des Productions bovines et chevalines

* INRA Station de Recherches

sur la Viande

Theix 63122 Saint-Genès-Champanelle

\section{Engraissement des vaches de réforme de race Limousine}

\begin{abstract}
A l'échelon national, plus de $40 \%$ de la viande dite de boeuf provient de l'abattage de vaches de réforme. Dans les races à viande, les vaches sont le plus souvent maigres lorsqu'elles sont réformées. Une phase d'engraissement est nécessaire pour bien valoriser ces animaux qui peuvent reprendre 80 à $100 \mathrm{~kg}$ de poids vif et fournir des carcasses de très bonne qualité.
\end{abstract}

La vache de réforme représente une part importante du produit brut du troupeau bovin : 10 à $15 \%$ pour les élevages spécialisés dans la production laitière et de 20 à $30 \%$ - selon les systèmes de production - pour ceux spécialisés dans la production de viande. Ainsi, dans cer-

\section{Résumé}

L'évolution de la reprise de poids et la composition corporelle de vaches de réforme limousines ont été étudiées en fonction de la durée d'engraissement, des apports alimentaires et de l'âge des animaux.

Lorsqu'elles sont alimentées avec des régimes d'ingestibilité et de concentration énergétique élevées (ensilage de maïs et concentré), les vaches de réforme peuvent, malgré leur faible capacité d'ingestion $(1,85$ à $1,90 \mathrm{~kg}$ MS / $100 \mathrm{~kg}$ poids vif), être engraissées en 2 mois, 2 mois et demi, et réaliser un gain de poids journalier de l'ordre de $1200 \mathrm{~g}$. Leur gain de carcasse est important, de l'ordre de 50 $\mathrm{kg}$, et la quantité de muscle dans ce gain est voisine de $20 \mathrm{~kg}$. Au-delà de cette durée d'engraissement, la reprise de poids devient beaucoup plus lente et le gain de carcasse est essentiellement constitué de dépôts adipeux.

L'efficacité alimentaire diminue tout au long de la période d'engraissement, mais cette diminution devient plus rapide au-delà des 2 premiers mois. L'efficacité chute également de façon importante lorsque les apports alimentaires sont limités. Il ne paraît donc pas intéressant de restreindre l'alimentation des animaux au cours de leur période de finition. En effet, les restrictions alimentaires conduisent à des vitesses de reprise de poids très faibles, et il est alors nécessaire de prolonger de façon trop importante la période d'engraissement.

Dans les conditions de notre étude, réalisée sur des vaches très âgées, un accroissement du niveau d'apports azotés n'a eu aucun effet sur la reprise de poids et la composition des carcasses.

L'analyse des performances en fonction de l'âge des animaux au moment de leur réforme montre que la capacité de reprise de poids des vaches, et donc leur gain de carcasse, sont d'autant plus élevés qu'elles sont jeunes. Toutefois, les vaches de race Limousine sont capables de produire jusqu'à l'âge de 13 ans des carcasses de poids et de composition très satisfaisants. Seules les vaches très âgées, de 14 ans et plus, sont difficiles à engraisser. taines races comme la Limousine et la Charolaise, cette vache a une valeur commerciale élevée lorsqu'elle est correctement finie et son engraissement se justifie encore plus qu'en race laitière. Toutefois, la conduite de l'engraissement reste empirique et ne donne pas toujours de bons résultats (carcasses trop grasses et/ou trop légères). Ce phénomène est d'ailleurs accentué depuis peu avec l'interdiction d'utiliser les anabolisants, qui se révélaient très efficaces et permettaient en outre de réduire la variabilité de la qualité de carcasse de ces animaux. En effet, les vaches de réforme sont des animaux d'âge, de format, d'état corporel, d'état sanitaire et de stade de gestation très variés.

Des études descriptives ont été réalisées depuis longtemps sur les vaches de réforme de races Normande et Pie-Noire (Béranger et Malterre 1968, Béranger et al 1970, Malterre 1972, Robelin et Lestrade 1982, Colleau et al 1984) et, plus récemment, sur des vaches de race Salers (Malterre 1986). En revanche, très peu de travaux ont été effectués sur les vaches de réforme de race à viande (essais de l'ITEB cités par Malterre 1986), alors que l'on peut penser que les animaux de race spécialisée à viande sont capables de reprendre plus de muscle et de réaliser des gains de poids plus importants que les animaux des races laitières. C'est pourquoi il a paru nécessaire de mettre en place des études spécifiques visant à mieux connaître et exploiter de manière optimale la capacité de reprise de poids des vaches de réforme de race Limousine lorsqu'elles sont engraissées après tarissement au cours de l'hiver.

Ces études ont été conduites au Centre INRA de Clermont-Ferrand - Theix entre 1985 et 
Tableau 1. Caractéristiques des animaux en début d'expérience.

\begin{tabular}{|c|c|c|c|}
\hline LOT & $\begin{array}{c}1 \\
\text { Maigre }\end{array}$ & $\stackrel{2}{\underset{\substack{\text { Engraissement } \\
\text { normal }}}{\operatorname{con}}}$ & $\begin{array}{c}3 \\
\underset{\text { prolongé }}{\text { Engraissement }}\end{array}$ \\
\hline $\begin{array}{l}\text { Nombre d'animaux } \\
\text { Poids initial }(\mathrm{kg}) \\
\text { Note d'état corporel }(/ 5) \\
\text { Taille des adipocytes }(\mu) \\
\text { Age des animaux (années) }\end{array}$ & $\begin{array}{c}5 \\
522 \\
1,2 \\
41 \\
6,8\end{array}$ & $\begin{array}{c}5 \\
508 \\
1,3 \\
40 \\
7,8\end{array}$ & $\begin{array}{c}5 \\
520 \\
1,3 \\
40 \\
6,8\end{array}$ \\
\hline
\end{tabular}

1988. Au cours de 3 campagnes d'expérimentations successives, nous avons analysé l'évolution de la reprise de poids et de la composition corporelle ainsi que celle de la capacité d'ingestion de vaches maigres, taries, engraissées avec un régime alimentaire bien connu à base d'ensilage de maïs.

Nous avons également étudié l'influence du niveau des apports énergétiques, du niveau d'apports azotés et de l'âge des animaux sur la vitesse de reprise de poids et la composition de la carcasse.

\section{1 / Conditions expérimentales}

La majeure partie des animaux a été achetée à un groupement de producteurs qui s'est approvisionné principalement sur le département de la Haute-Vienne. Les vaches devaient correspondre aux caractéristiques suivantes: avoir fait 3 lactations au minimum, être vides et taries, être en bon état sanitaire et être maigres à l'achat en étant restées le plus longtemps possible avec leur veau.

L'ensemble des animaux a été engraissé à Theix en stabulation entravée. Au cours d'une période dite d'adaptation, de 3 semaines environ, les vaches ont été systématiquement déparasitées et ont fait l'objet de pesées régulières dont une double pesée en fin de période sur un même régime alimentaire afin de procéder à la mise en lots. On a, en outre, déterminé leur état d'engraissement par maniements féchelle de notes comprises entre 0 et 5) et par la mesure du diamètre des adipocytes du tissu adipeux sous-cutané caudal (Robelin et Agabriel 1986).

$\mathrm{Au}$ cours de la période expérimentale, les animaux ont été pesés régulièrement chaque semaine et les quantités ingérées ont été contrôlées individuellement 5 jours par semaine. Les aliments ont été analysés chimiquement, l'ensilage de maïs a fait l'objet d'une mesure de digestibilité sur bovins.

A l'abattage les carcasses et les différents éléments du $5^{\circ}$ quartier (dépôts adipeux notamment) ont été pesés, les rendements en carcasse (commercial et vrai) ont été déterminés et la $6^{\mathrm{e}}$ côte a été prélevée afin d'estimer, à partir de sa composition, celle de la carcasse.

Dans le cadre de la première expérience, on a effectué la dissection complète de 2 groupes de 5 animaux, représentatifs des vaches respectivement en début et en fin de période de fini- tion. Au cours de cette dissection, on a mesuré le poids de tous les organes, celui des différents tissus de la carcasse et du $5^{\circ}$ quartier (squelette, muscles, tissus adipeux). On a également mesuré le poids des différentes régions musculaires et des différents tissus adipeux (souscutané, intermusculaire, interne, omental, mésentérique et périrénal). Enfin, tous ces tissus ont été broyés et ont fait l'objet d'une analyse chimique (teneurs en eau, lipides, protéines, minéraux, et valeur calorifique). L'ensemble de ces résultats a permis de déterminer la composition chimique du corps entier. Les résultats détaillés de ces mesures font l'objet d'une publication spécifique (Robelin, Agabriel, Malterre et Bonnemaire, à paraître).

Après dissection de chaque carcasse, les muscles Long Dorsal (muscle «tendre») et Pectoral Profond (muscle " dur») ont fait l'objet de mesures chimiques - teneurs en matière sèche et en collagène, solubilité du collagène (Kopp et Bonnet 1982) - et physiques : détermination de la force de compression pour un taux de déformation de $80 \%$ (Lepetit 1982, Lepetit et Culioli 1986).

\section{Expérience 1}

L'état d'engraissement des 29 vaches achetées étant très variable (notes d'état corporel comprises entre 0,5 et 3,5 ), trois lots de vaches ont été constitués en fonction de leur état initial. Selon leur lot d'appartenance les vaches ont alors été plus ou moins sous-alimentées (foin + paille en quantités très limitées) au cours d'une période de 55 jours afin d'homogénéiser leur état d'engraissement et de réduire celui des vaches les plus grasses à l'achat (notes d'état comprises entre 0,7 et 2,7 après 55 jours d'homogénéisation). A la fin de cette période, 3 lots de 5 animaux les plus comparables possibles ont été constitués sur la base de leur poids vif, de leur état d'engraissement, de leur âge et de leur format (tableau 1).

- Le $1^{\text {er }}$ lot a été abattu «maigre» avant engraissement et une demi-carcasse de chaque animal a été disséquée et analysée.

- Les $2^{\mathrm{e}}$ et $3^{\text {e }}$ lots ont été engraissés respectivement pendant 79 (engraissement normal) et 123 jours (engraissement prolongé). Les vaches de ces 2 lots ont reçu à volonté un régime à base d'ensilage de maïs complémenté quotidiennement avec $0,5 \mathrm{~kg}$ de tourteau de soja, 0,5 $\mathrm{kg}$ de maïs grain, $100 \mathrm{~g}$ d'urée et $120 \mathrm{~g}$ de complément minéral vitaminé (CMV). Les carcasses de 5 vaches du lot 3 ont en outre été disséquées et analysées. 


\section{Expérience 2}

Trois lots de 10 vaches comparables ont été constitués sur la base de leur poids vif, de leur état d'engraissement et de leur âge. Au cours de la période expérimentale les animaux d'un même lot ont toujours reçu la même ration journalière.

- Lot 1 (niveau d'alimentation élevé) : ensilage de maïs à volonté, $0,5 \mathrm{~kg}$ de tourteau de soja, 2,0 kg de maïs grain, $100 \mathrm{~g}$ d'urée et 120 à $150 \mathrm{~g}$ de CMV.

- Lot 2 (niveau d'alimentation moyen) : $25 \mathrm{~kg}$ d'ensilage de maïs, $0,5 \mathrm{~kg}$ de tourteau de soja, $1,0 \mathrm{~kg}$ de maïs grain, $80 \mathrm{~g}$ d'urée et 120 à $150 \mathrm{~g}$ de CMV.

- Lot 3 (niveau d'alimentation faible) : $21 \mathrm{~kg}$ d'ensilage de maïs, $0,5 \mathrm{~kg}$ de tourteau de soja, $0,5 \mathrm{~kg}$ de maïs grain, $50 \mathrm{~g}$ d'urée et 120 à $150 \mathrm{~g}$ de CMV.

La durée de la période expérimentale a été variable selon les lots, l'objectif étant de commercialiser les animaux à même poids final.

\section{Expérience 3}

Trente vaches parmi 33 ont servi à la constitution de 3 lots de vaches comparables quant à leur poids vif, leur âge et leur état d'engraissement. Chaque lot a alors reçu des niveaux d'apports d'azote différents de telle sorte que le niveau le plus faible corresponde à celui des apports recommandés pour les vaches de réforme adultes de race à viande (soit environ $90 \mathrm{~g}$ PDI/UFL, INRA 1988) et les deux autres à 110 et $130 \%$ de ce niveau de référence.

Ces différents niveaux d'apports azotés ont été réalisés en faisant varier la composition de l'aliment concentré qui représentait entre 24 et $28 \%$ de la matière sèche de la ration totale à base d'ensilage de maïs.

Les animaux d'un même lot ont donc reçu la même ration journalière au cours de la période expérimentale dont la durée a été identique pour les 3 lots. Outre de l'ensilage de maïs à volonté, $130 \mathrm{~g}$ d'urée et $120 \mathrm{~g}$ de CMV, les vaches du lot 1 (niveau de complémentation en azote "normal ") ont reçu $0,5 \mathrm{~kg}$ de tourteau de soja et $2,3 \mathrm{~kg}$ de maïs grain, celles du lot 2 (niveau de complémentation en azote « inter-

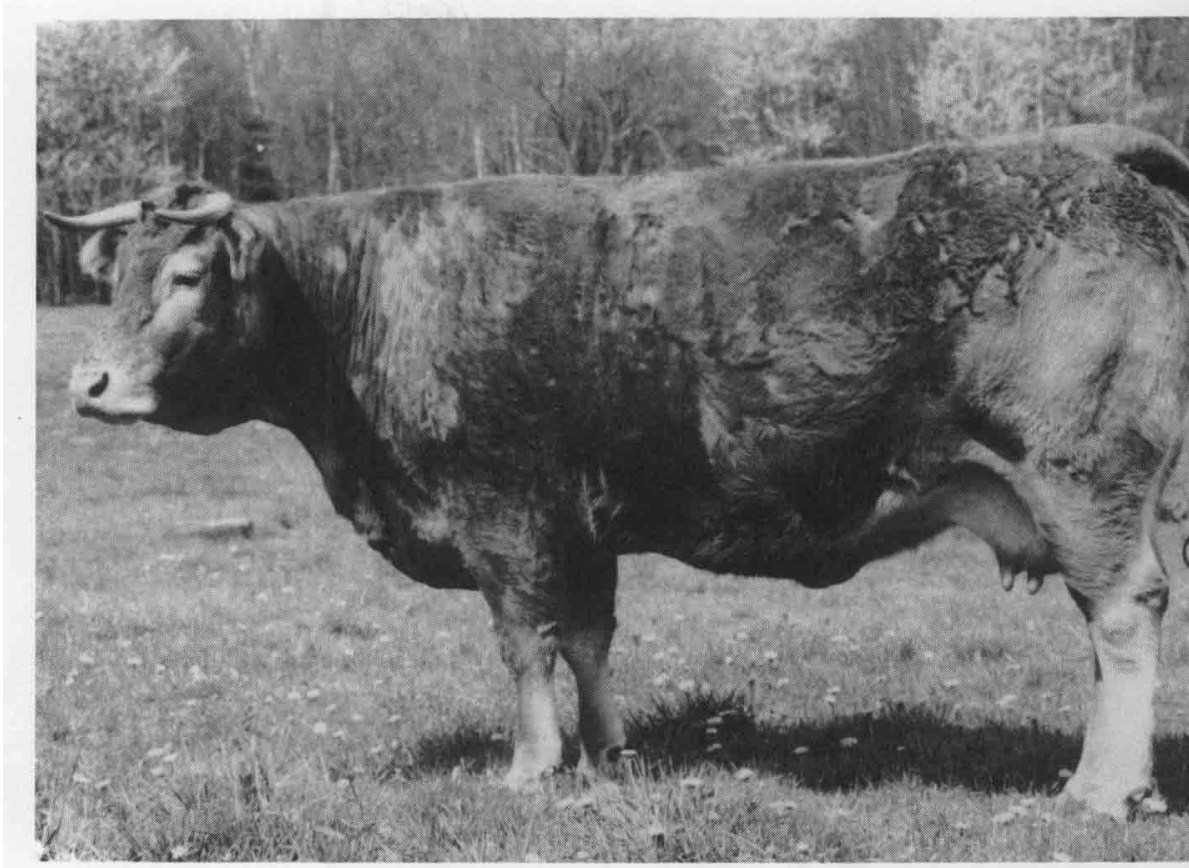

médiaire ») $1,5 \mathrm{~kg}$ de tourteau de soja et $1,4 \mathrm{~kg}$ de maïs grain, tandis que celles du lot 3 (niveau de complémentation en azote "élevé») ne recevaient que du tourteau de soja, à raison de $3 \mathrm{~kg} /$ jour. Dans les 3 lots, l'apport d'énergie par le concentré a été identique.

\section{2 / Résultats}

\section{1 / Evolution du poids vif et de la capacité d'ingestion au cours de la période d'engraissement}

Les vaches engraissées durant 79 jours ont consommé en moyenne $10,6 \mathrm{~kg}$ de matière sèche par jour pour une vitesse de reprise de poids de $1230 \mathrm{~g} / \mathrm{j}$ (tableau 2). Elles ont donc ingéré en moyenne $8,6 \mathrm{~kg}$ de matière sèche d'une ration comportant $90 \%$ d'ensilage de maïs et seulement $10 \%$ de concentré, pour réaliser un $\mathrm{kg}$ de gain de poids vif, soit environ 7,5 $\mathrm{UFL} / \mathrm{kg}$ de gain.

Tableau 2. Performances d'engraissement des vaches des lots 2 (engraissement normal) et 3 (engraissement prolongé).

\begin{tabular}{|l|c|c|}
\hline \multicolumn{1}{|c|}{ Lot } & $\mathbf{2}$ & $\mathbf{3}$ \\
\hline \multicolumn{1}{|c|}{ Durée d'engraissement (j) } & $\mathbf{7 9}$ & $\mathbf{1 2 3}$ \\
Quantités ingérées & & 9,6 \\
- kg MS/jour & 10,6 & 10,2 \\
- kg MS/kg de gain & 8,6 & $118 \pm 10$ \\
\hline Reprise de poids (kg) & $97 \pm 12$ & 955 \\
Gain de poids vif (g/j) & 1230 & 4,8 \\
\hline Etat d'engraissement final & & 124 \\
- note sur 5 (maniements) & 4,3 & \\
- taille des adipocytes $(\boldsymbol{\mu})$ & 105 & \\
\hline
\end{tabular}

\section{Les vaches \\ Limousines se \\ caractérisent par \\ une faible capacité d'ingestion mais une bonne efficacité alimentaire.}

La viande de vaches de réfor représente plus de $80 \%$ de 1 viande rouge issue des anim adultes du troupeau Limous. 
Tableau 3. Résultats d'abattage.

\begin{tabular}{|l|c|c|c|}
\hline Lot & $\mathbf{1}$ & $\mathbf{2}$ & $\mathbf{3}$ \\
Durée d'engraissement $(\mathrm{j})$ & 0 & 79 & 123 \\
Poids vif avant abattage $(\mathrm{kg})$ & 522 & 605 & 638 \\
\hline Poids vif vide $(\mathrm{kg})$ & $452 \mathrm{a}$ & $533 \mathrm{~b}$ & $558 \mathrm{~b}$ \\
Poids de carcasse froide $(\mathrm{kg})$ & $297 \mathrm{a}$ & $344 \mathrm{~b}$ & $364 \mathrm{~b}$ \\
\hline Rendement économique $(\%)^{*}$ & 56,9 & 56,9 & 56,9 \\
Rendement vrai $(\%)^{\star \star}$ & $67,3 \mathrm{a}$ & $65,8 \mathrm{a}$ & $\mathbf{6 6 , 5 \mathrm { a }}$ \\
Dépôts adipeux carcasse $(\%)$ & 8,6 & $17,7^{(1)}$ & 21,0 \\
\hline
\end{tabular}

Sur une même ligne, les valeurs suivies de lettres différentes sont significativement différentes à $P<0,01$.

* Poids de carcasse froide / poids vif avant abattage.

** Poids de carcasse chaude / poids vif vide.

"' Estimés.

Figure 1. Evolution du poids des vaches au cours de la période d'engraissement.

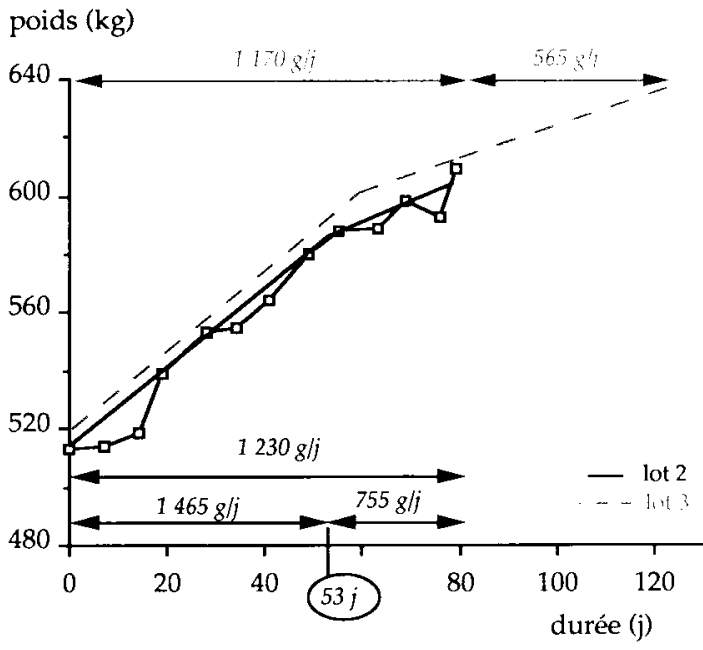

Figure 2. Evolution des quantités ingérées au cours de la période d'engraissement (vaches du lot 2 : engraissement de durée normale).

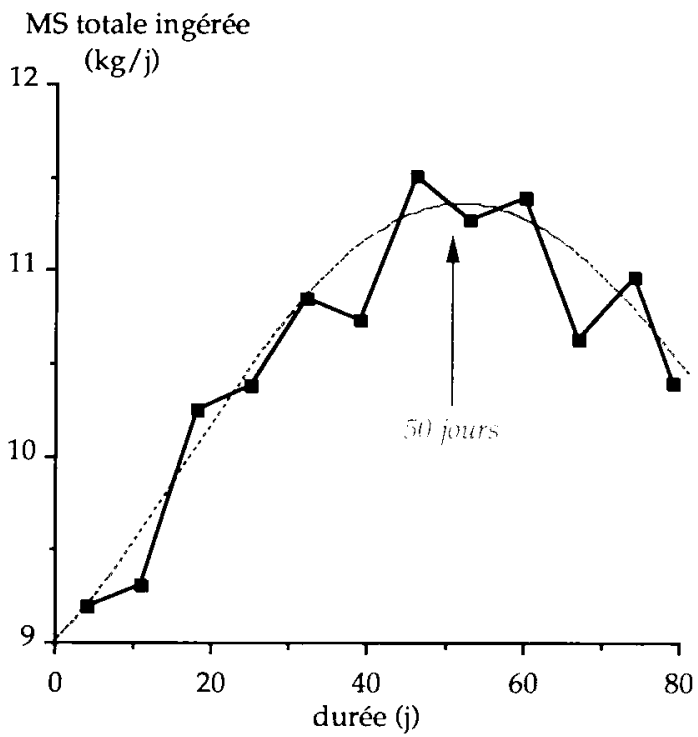

Ces résultats moyens correspondent toutefois à des évolutions très importantes au cours des 79 jours d'engraissement. En effet, la reprise de poids des animaux a été élevée durant les 53 premiers jours puis a fortement baissé : successivement 1465 puis $755 \mathrm{~g} / \mathrm{j}$ (figure 1). Parallèlement, les quantités ingérées par les animaux ont augmenté régulièrement jusqu'au 50ème jour d'engraissement $(11,5 \mathrm{~kg} \mathrm{MS} / \mathrm{j}$ au maximum) et ont diminué par la suite (figure 2).

L'indice de consommation (quantités ingérées / vitesse de reprise de poids vif) a augmenté tout au long de la période d'engraissement. Toutefois, cet accroissement a été beaucoup plus rapide à partir du 50ème jour d'engraissement car la diminution des consommations a été nettement moins importante que celle du gain de poids des animaux (figure 3).

Les vaches dont l'engraissement a été prolongé jusqu'à 123 jours (lot 3) peuvent être comparées à celles du lot 2 au cours des 79 premiers jours d'engraissement. Au cours de cette période les 5 vaches du lot 3 ont consommé 9,9 $\mathrm{kg} \mathrm{MS} / \mathrm{j}$ pour un gain de poids journalier de

Figure 3. Evolution de lindice de consommation ( $k g$ MS ingérée / kg de gain de poids vif) au cours de la période d'engraissement.

indice de consommation cumulé

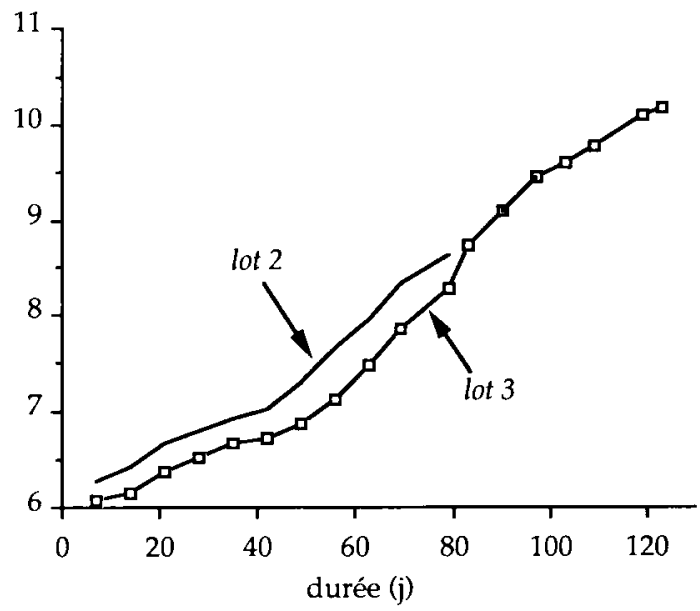


$1170 \mathrm{~g}$. Elles ont donc ingéré 8,4 kg MS par kg de gain de poids vif, ce qui correspond à une efficacité alimentaire très voisine de celle observée pour le lot 2 qui a consommé $8,6 \mathrm{~kg}$ $\mathrm{MS} / \mathrm{kg}$ de gain). De plus, leur état d'engraissement estimé in vivo à 79 jours d'engraissement a été tout à fait proche de celui des animaux du lot 2 : notes de 3,9 et 4,3 ; et diamètre moyen des adipocytes de 117 et $105 \mu$ respectivement pour les lots 3 et 2 .

En revanche, au cours des 44 jours suivants, on a pu mettre en évidence une chute des quantités journalières ingérées ainsi que de la reprise de poids des animaux (gain de poids de $565 \mathrm{~g} / \mathrm{j}$ vs $1170 \mathrm{~g} / \mathrm{j}$ pour la période précédente, figure 1). L'indice de consommation a augmenté très rapidement au cours de cette période (figure 3).

Globalement, au cours des 123 jours d'engraissement (tableau 2) les animaux ont consommé $9,6 \mathrm{~kg} \mathrm{MS} / \mathrm{j}$ et réalisé un croît de seulement $955 \mathrm{~g} / \mathrm{j}$. Ils ont donc été beaucoup moins efficaces que les animaux du lot 2, leur indice de consommation étant de $20 \%$ supérieur (10,2 contre $8,4 \mathrm{~kg} \mathrm{MS} / \mathrm{kg}$ de gain).

\section{2 / Résultats d'abattage}

\section{a / Poids et composition des carcasses}

D'un poids et d'un état d'engraissement comparables à ceux des animaux destinés à être engraissés, les 5 vaches abattues maigres ont produit des carcasses de $297 \mathrm{~kg}$ comportant 26 $\mathrm{kg}$ de dépôts adipeux.

Les animaux engraissés pendant 79 jours ont produit des carcasses d'un poids moyen de 344 $\mathrm{kg}$ dont le pourcentage de gras estimé à partir de la composition de la 6ème côte et des dépôts adipeux du 5ème quartier (Robelin, communication personnelle) a été de $17,7 \%$ (Lableau 3), soil environ $60 \mathrm{~kg}$ de dépôts adipeux. La quantité de gras est donc plus de 2 fois supérieure à celle des vaches abattues « maigres » en début d'expérience.

A l'abattage, les animaux du lot engraissé pendant 123 jours étaient plus gras, tant au niveau de la note d'élat et de la taille des adipo- cytes qu'au niveau de la composition réelle de leurs carcasses. D'un poids moyen final plus élevé que les vaches du lot 2 (638 contre 605 $\mathrm{kg}$ ), du fait de leur durée d'engraissement plus longue, les animaux de ce lot ont produit des carcasses plus lourdes $(+20 \mathrm{~kg})$ mais aussi plus grasses (tableau 3 ).

Les compositions anatomiques des carcasses des vaches des lots 1 et 3, déterminées après dissection, figurent au tableau 4 .

Ces résultats permettent de mettre clairement en évidence que les vaches de réforme de race Limousine sont capables d'accroître leur musculature d'environ $20 \mathrm{~kg}$ au cours de la période d'engraissement qui précède leur abattage (figure 4). En réalité il n'est pas nécessaire de

Figure 4. Composition des carcasses des vaches des lots 1 (maigre) et 3 (engraissement prolongé) obtenue par dissection.

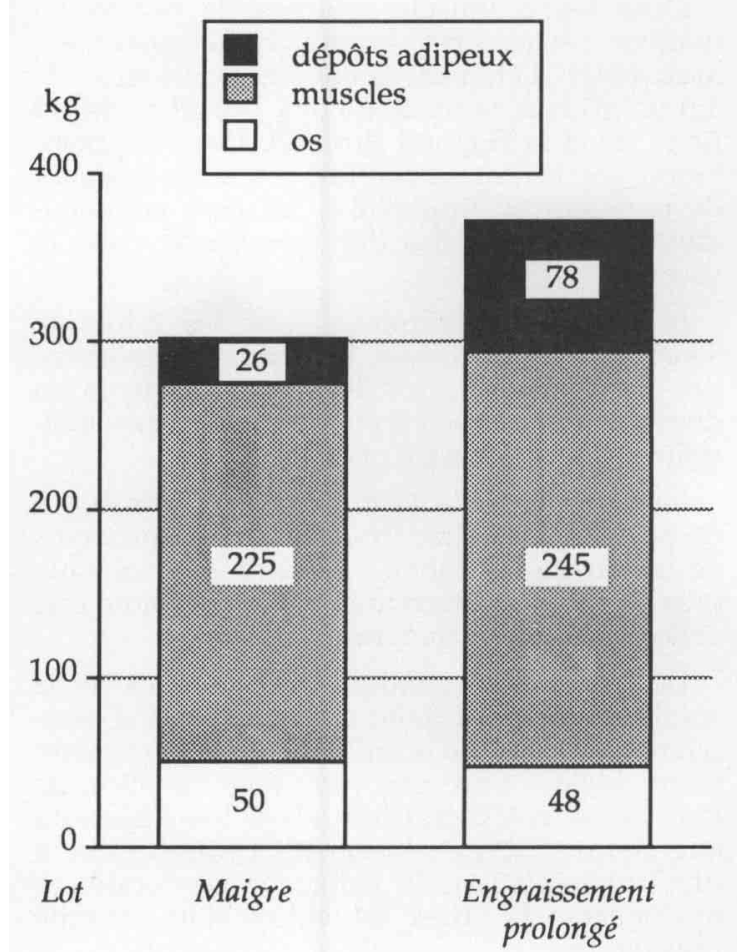

Tableau 4. Composition anatomique des carcasses de vaches maigres (lot 1) et grasses (lot 3 = engraissement prolongé).

\begin{tabular}{|c|c|c|}
\hline LOT & 1 & 3 \\
\hline $\begin{array}{l}\text { Poids vif vide }(\mathrm{kg}) \\
\text { Dépôts adipeux tolaux }(\mathrm{kg}) \\
\text { Squelette entier }(\mathrm{kg})\end{array}$ & $\begin{array}{l}452 \pm 16 \\
34 \pm 5 \\
62 \pm 4\end{array}$ & $\begin{array}{c}557 \pm 19 \\
106 \pm 6 \\
60 \pm 3\end{array}$ \\
\hline $\begin{array}{l}\text { Carcasse } \\
\text { - poids chaud }(\mathrm{kg}) \\
\text { - dépôts adipeux }(\mathrm{kg}) \\
\text { - muscles }(\mathrm{kg}) \\
\text { - os }(\mathrm{kg})\end{array}$ & $\begin{array}{r}304 \pm 9 \\
26 \pm 4 \\
225 \pm 3 \\
50 \pm 4\end{array}$ & $\begin{aligned} 372 & \pm 16 \\
78 & \pm 7 \\
245 & \pm 8 \\
48 & \pm 3\end{aligned}$ \\
\hline $\begin{array}{l}\text { Muscles } \\
\text { - poids des muscles de la cuisse }(\mathrm{kg}) \\
\text { - poids des muscles dorsaux }(\mathrm{kg}) \\
\text { - poids du psoas* }(\mathrm{kg}) \\
\text { - poids du long dorsal }{ }^{* *}(\mathrm{~kg})\end{array}$ & $\begin{array}{r}83,4 \pm 0,2 \\
30,3 \pm 0,5 \\
3,3 \pm 0,1 \\
8,2 \pm 0,2\end{array}$ & $\begin{array}{r}87,6 \pm 1,9 \\
32,4 \pm 1,3 \\
3,1 \pm 0,2 \\
8,9 \pm 0,3\end{array}$ \\
\hline
\end{tabular}

\footnotetext{
* Psoas ou filet.

** I long dorsal ou faux-filet.
}

Au bout de 80 jours, le gain de carcasse est de $50 \mathrm{~kg}$ dont $20 \mathrm{~kg}$ de muscles. Au-delà de cette période, le gain supplémentaire est essentiellement constitué de gras. 
prolonger la période de finition des vaches audelà de 2 mois et demi pour leur permettre une telle reprise musculaire. En effet, si l'on calcule la quantité de dépôt adipeux de la carcasse des vaches du lot 2 engraissées pendant 79 jours, on peut mettre en évidence qu'elle est passée de 26 à 60 puis $78 \mathrm{~kg}$ pour les stades 0,79 et 123 jours d'engraissement. Cela montre bien que les $20 \mathrm{~kg}$ de carcasse supplémentaires obtenus en prolongeant l'engraissement de 44 jours sont essentiellement constitués de gras et donc que la quasi totalité du gain de muscle s'est effectuée au cours des 79 premiers jours d'engraissement.

Dans ce gain de muscle, on ne constate pas d'accroissement préférentiel des muscles de valeur bouchère élevée ( $+4 \mathrm{~kg}$ au niveau de la cuisse, $+2 \mathrm{~kg}$ au niveau du dos).

\section{b / Caractéristiques physico-chimiques des muscles}

Dans les 2 muscles étudiés, la teneur en matière sèche s'est accrue significativement avec l'état d'engraissement des animaux: de 1,0 point dans le muscle Long Dorsal et de 1,9 point dans le Pectoral Profond. Cette augmentation est certainement liée à l'accroissement de la teneur en lipides des muscles, concomitant à l'élévation des dépôts adipeux dans la carcasse.

Bien que les différences entre les 2 lots ne soient pas significatives, la teneur en collagène des 2 muscles s'accroît légèrement pour le lot dont l'engraissement a été prolongé, particulièrement dans le muscle pectoral.

Les variations de la force de compression à $80 \%$ de déformation traduisent les variations de teneur en collagène, la viande des animaux gras se révélant légèrement plus résistante que celle des animaux maigres.

De très fortes variations individuelles de la solubilité du collagène intramusculaire masquent une éventuelle influence de l'engraissement. Malgré tout, une forte augmentation de solubilité est constatée chez les animaux engraissés. Cette évolution est peut-être due à une augmentation du collagène néoformé, ce qui pourrait favoriser l'obtention d'une viande plus tendre.

\section{3 / Influence du niveau d'apports énergétiques (tableau 6)}

Comme le prévoyait le protocole expérimental les différences de niveau d'alimentation entre lots ont été importantes, ces niveaux devant permettre des vitesses de reprise de poids vif de 1200 , 900 et $600 \mathrm{~g}$ par jour. Alimentées à volonté, les vaches du lot 1 ont consommé $10,5 \mathrm{~kg}$ de matière sèche par jour, soit une quantité très voisine de celle mesurée au cours de la première expérience $(10,6 \mathrm{~kg}$ $\mathrm{MS} / \mathrm{j}$ ) avec des animaux dont le poids moyen était très comparable. Les quantités ingérées par les vaches des lots 2 et 3 ont été en moyenne de 8,7 et $7,0 \mathrm{~kg} \mathrm{MS} / \mathrm{j}$ ce qui correspond à une réduction du niveau des apports énergétiques de 19 et $35 \%$ respectivement.

Figure 5. Evolution du poids des vaches en fonction du niveau d'apports énergétiques.

poids vif $(\mathrm{kg})$

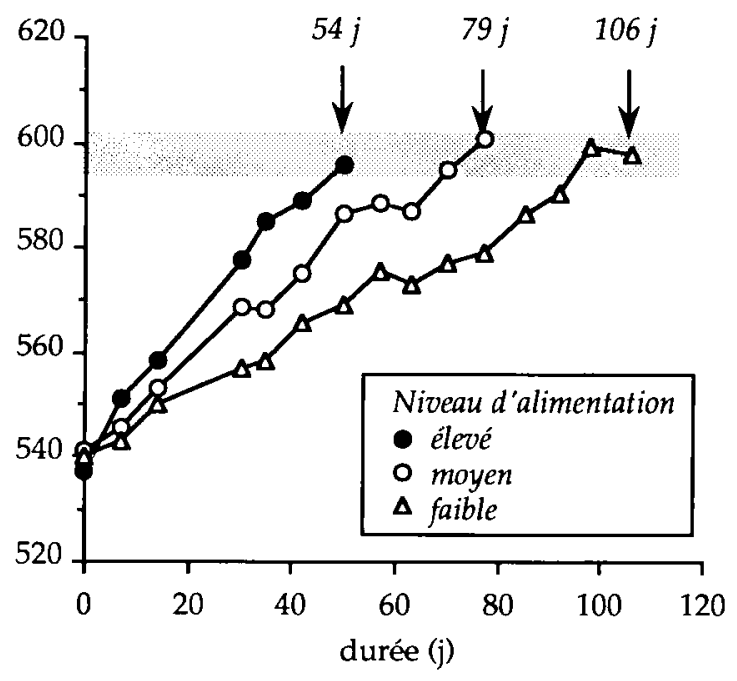

Tableau 5. Caractéristiques physico-chimiques des muscles Long dorsal et Pectoral profond des vaches maigres (lot 1) et grasses (lot 3).

\begin{tabular}{|c|c|c|}
\hline $\begin{array}{c}\text { Lot } \\
\text { Nombre de vaches }\end{array}$ & $\begin{array}{l}1 \\
5\end{array}$ & $\begin{array}{l}\mathbf{3} \\
\mathbf{5}\end{array}$ \\
\hline $\begin{array}{l}\text { Muscle long dorsal } \\
\text { - matière sèche }(\%) \\
\text { - teneur en collagène (mg/g frais) } \\
\text { - solubilité du collagène }(\%) \\
\text { - force de compression }(\mathrm{N} / \mathrm{cm} 2) \\
\text { (taux de déformation }=80 \%)\end{array}$ & $\begin{array}{r}24,5 \pm 0,8 \\
4,4 \pm 0,6 \\
9,3 \pm 5,6 \\
29,9 \pm 8,1\end{array}$ & $\begin{array}{r}25,5 \pm 0,5 \\
4,5 \pm 0,6 \\
20,3 \pm 11,6 \\
41,1 \pm 15,9\end{array}$ \\
\hline $\begin{array}{l}\text { Muscle pectoral profond } \\
\text { - matière sèche }(\%) \\
\text { - teneur en collagène (mg/g frais) } \\
\text { - solubilité du collagène }(\%) \\
\text { - force de compression }(\mathrm{N} / \mathrm{cm} 2) \\
\text { (taux de déformation }=80 \%)\end{array}$ & $\begin{array}{r}24,3 \pm 0,8 \\
10,8 \pm 2,1 \\
10,9 \pm 9,5 \\
113,1 \pm 30,6\end{array}$ & $\begin{array}{r}26,2 \pm 1,5 \\
11,9 \pm 2,1 \\
22,3 \pm 9,5 \\
130,6 \pm 36,3\end{array}$ \\
\hline
\end{tabular}


Tableau 6. Influence du niveau d'apports énergétiques sur les performances d'engraissement des vaches de réforme.

\begin{tabular}{|c|c|c|c|}
\hline $\begin{array}{c}\text { Lot } \\
\text { Niveau d'alimentation }\end{array}$ & $\begin{array}{l}1 \\
\text { élevé }\end{array}$ & $\stackrel{2}{\text { moyen }}$ & $\begin{array}{c}3 \\
\text { faible }\end{array}$ \\
\hline $\begin{array}{l}\text { Nombre d'animaux } \\
\text { Age moyen (années) } \\
\text { Etat d'engraissement initial } \\
\text { - note sur } 5 \\
\text { - diamètre des adipocytes }(\mu)\end{array}$ & $\begin{array}{c}10 \\
8,4\end{array}$ & $\begin{array}{c}9 \\
8,3 \\
1,5 \\
45\end{array}$ & $\begin{array}{c}9 \\
9,1 \\
\\
1,4 \\
44 \\
\end{array}$ \\
\hline $\begin{array}{l}\text { Quantités ou énergie ingérées } \\
\text { - kg MS/j } \\
\text { - UFL/j } \\
\text { - UFL/kg de gain } \\
\text { Résultats d'engraissement } \\
\text { - poids initial (kg) } \\
\text { - poids final }(\mathrm{kg}) \\
\text { - durée (j) } \\
\text { - gain de poids vif }(\mathrm{g} / \mathrm{j}) \\
\text { - note d'état finale }(/ 5)\end{array}$ & $\begin{array}{c}537 \\
601 \\
54 \\
1200 \\
4,1\end{array}$ & $\begin{array}{c}541 \\
606 \\
79 \\
815 \\
4,2\end{array}$ & $\begin{array}{l}540 \\
598 \\
106 \\
545 \\
3,8\end{array}$ \\
\hline $\begin{array}{l}\text { Résultats d'abattage } \\
\text { - poids vif vide }(\mathrm{kg}) \\
\text { - poids de carcasse froide }(\mathrm{kg}) \\
\text { - rendement économique }(\%)^{*} \\
\text { - rendement vrai }(\%)^{* *} \\
\text { - dépôts adipeux totaux }(\mathrm{kg})^{(1)} \\
\text { - dépôts adipeux totaux } \\
\text { (en \% du poids vif vide) }\end{array}$ & $\begin{array}{c}535 \pm 34 \\
351 \pm 35 \\
58,5 \\
66,7 \\
84 \pm 18 \\
15,7 \pm 2,7\end{array}$ & $\begin{array}{c}531 \pm 31 \\
344 \pm 23 \\
56,8 \\
65,5 \\
79 \pm 15 \\
14,9 \pm 2,9\end{array}$ & $\begin{array}{c}525 \pm 23 \\
346 \pm 21 \\
57,9 \\
67,5 \\
74 \pm 13 \\
14,1 \pm 2,2\end{array}$ \\
\hline
\end{tabular}

* Poids de carcasse froide / poids vil avant abattage.

** Poids de carcasse chaude / poids vif vide.

(1) Estimés.
La réduction du niveau alimentaire présente peu d'intérêt : allongement de la durée d'engraissement sans amélioration de la qualité des carcasses.
La modulation du niveau d'alimentation s'est traduite par des différences très importantes de la vitesse de reprise de poids : $1200 \mathrm{~g} / \mathrm{j}$ pour le lot 1 alimenté à volonté, un peu plus de $800 \mathrm{~g} / \mathrm{j}$ pour le lot 2 et seulement $550 \mathrm{~g} / \mathrm{j}$ pour le lot 3 qui a été le plus limité. Par conséquent, les animaux qui ont été limités ont dû être engraissés pendant une période plus longue que ceux alimentés à volonté afin de réaliser des gains de poids globaux comparables entre lots. Ainsi, les vaches du lot 3 dont la vitesse de reprise de poids a été approximativement 2 fois plus faible que celle des animaux du lot 1 , ont été engraissées 2 fois plus longtemps que ces derniers (106 et 54 jours respectivement : figure 5).

L'indice de consommation, exprimé en UFL ingérées par $\mathrm{kg}$ de gain de poids (afin de tenir compte des différences de concentration énergétique entre régimes), a fortement augmenté avec la restriction alimentaire : +20 et $+43 \%$ respectivement pour les lots 2 et 3 comparativement au lot 1 . Cet accroissement très important de l'indice de consommation résulte du fait que la part des besoins d'entretien des animaux dans leurs besoins tolaux (entretien + gain de poids) devient de plus en plus importante lorsque la quantité d'énergie apportée à l'animal diminue.
Les animaux des différents lots ont été abattus à des poids vifs semblables. Leurs poids vifs vides et leurs poids de carcasses ont également été très semblables d'un lot à l'autre. En outre, les carcasses dont le poids moyen s'est situé entre 344 et $351 \mathrm{~kg}$ ont été jugées globalement satisfaisantes sur le plan commercial (état d'engraissement notamment). Il semble donc que le niveau d'alimentation (élevé, moyen ou faible) a peu influencé la qualité des carcasses produites. Seul l'état d'engraissement des vaches du lot le plus limité a été un peu plus faible: $-0,3$ ou 0,4 point sur la note d'état et -5 à -10 $\mathrm{kg}$ de dépôts adipeux totaux.

\section{4 / Influence du niveau d'apports azotés (tableau 7)}

Par suite de très nombreux problèmes survenus sur les animaux, dont l'âge moyen était supérieur à 12 ans, les résultats définitifs ne portent que sur 18 vaches. Huit vaches ont en effet très mal consommé l'ensilage de maïs (plus de $3 \mathrm{~kg}$ de matière sèche en dessous de la moyenne) en liaison avec une pathologie podale importante, une vache a vêlé en cours d'expérience et 3 vaches étaient pleines d'un foetus dont le poids à l'abattage était élevé $(22$, 
Tableau 7. Influence du niveau d'apports azotés sur les performances d'engraissement des vaches de réforme.

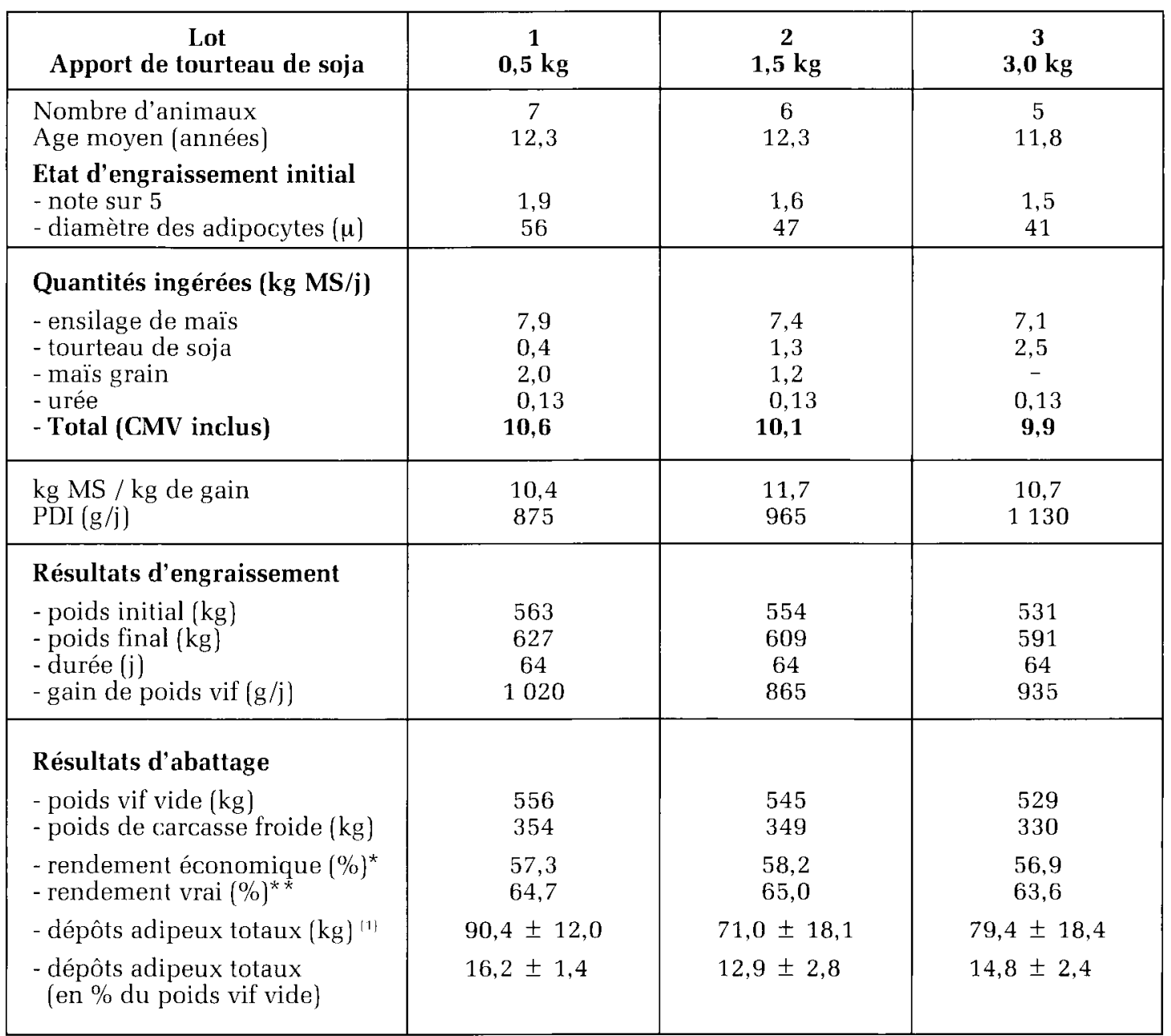

* Poids de carcasse froide/poids vif avant abatlage.

** Poids de carcasse chaude/poids vif vide

"1' Estimés.

32 et $33 \mathrm{~kg}$ ). Ces 12 vaches ont donc été éliminées des résultats.

Les quantités ingérées par les animaux du lot 1 (complémentation "normale" en azote, comparable à celle reçue par les animaux des lots témoins des deux expériences précédentes] ont été de $10,6 \mathrm{~kg} \mathrm{MS} / \mathrm{j}$ en moyenne, valeur identique à celles observées précédemment. En revanche, les vaches des lots 2 et 3 qui ont reçu pratiquement la même quantité de concentré que celles du lot 1 mais dont la proportion de tourteau de soja était beaucoup plus importante, ont consommé un peu moins d'ensilage de maïs. Cela s'est traduit par une diminution des quantités globales ingérées de 5 et $7 \%$ respectivement comparativement au lot 1 .

Les différences importantes d'apports azotés entre les 3 lots $(875,965$ et $1130 \mathrm{~g}$ de PDI par jour respectivement pour les lots 1,2 et 3) ne se sont pas traduites par des différences importantes dans le gain de poids vif des animaux. Globalement, les vitesses de reprise de poids observées sont modestes, ce qui est sans doute à relier à l'âge élevé des animaux. Paradoxale- ment, les performances les meilleures correspondent au lot le plus faiblement complémenté en azote

L'indice de consommation, exprimé en $\mathrm{kg}$ de matière sèche ingérée par kg de gain de poids, a été élevé dans tous les lots et compris entre 10,4 et 11,7 pour les 3 lots. Cela traduit le niveau de performance très moyen observé au cours de cette expérience.

A l'abattage, le poids moyen de carcasse de l'ensemble des animaux était de $345 \mathrm{~kg}$. Toutefois, les animaux du lot le plus fortement complémenté ont produit des carcasses plus légères. D'un poids plus faible en début d'expérience, à la suite d'une transition plus difficile, les animaux de ce lot ont en outre été pénalisés par un rendement en carcasse plus faible en moyenne. L'état d'engraissement moyen des carcasses a assez peu varié d'un lot à l'autre. Seul celui des animaux du lot 2 a été plus faible.

Dans les conditions de cette expérience et comparativement au niveau de référence (INRA 
1988), un apport supplémentaire de tourteau de soja n'a donc pas permis d'accroître les performances des animaux. Toutefois, il paraît difficile de conclure de façon définitive sur le niveau optimum d'apport d'azote à recommander pour des vaches de réforme de race Limousine. En effet, les animaux utilisés dans cette expérience étaient en moyenne très âgés (plus de 12 ans) et les effectifs par lot étaient très faibles.

\section{5 / Variations des performances liées à l'âge des animaux}

La capacité à reprendre du poids et à s'engraisser dépend de l'âge des vaches au moment de leur réforme (Malterre 1986, Malterre et Jones 1990). Nous avons donc analysé ce facteur à partir des résultats individuels des vaches qui, dans chacune des 3 expériences, ont reçu de l'ensilage de maïs à volonté et ont été engraissées durant une période normale comprise entre 50 et 80 jours. Les résultats sont présentés au tableau 8 , ils portent sur 49 animaux engraissés pendant 65 à 71 jours en moyenne selon les lots.

La vitesse de reprise de poids est d'autant plus faible que les vaches sont plus âgées et est de moins de $500 \mathrm{~g} / \mathrm{j}$ pour les animaux de 14 ans et plus. Toutefois, jusqu'à l'âge de 13 ans, cette diminution du gain de poids ne s'est pas traduite par une réduction du poids vif avant abattage ou du poids de carcasse des animaux car, jusqu'à cet âge, le poids des vaches en début d'engraissement était d'autant plus élevé que leur âge était avancé. Ainsi, malgré des différences de gain de poids importantes, les animaux âgés de 5 à 13 ans ont tous pesé (en moyenne par classe d'âge) $610 \mathrm{~kg}$ en fin d'engraissement et produit des carcasses d'un poids moyen de $345 \mathrm{~kg}$. Seuls les animaux les plus âgés (14 à 16 ans) ont produit des carcasses plus légères de $23 \mathrm{~kg}$.

La capacité d'ingestion des vaches varie relativement peu jusqu'à l'âge de 13 ans et est nettement plus faible chez les vaches plus âgées.

L'état d'engraissement des animaux en fin de période de finition et la composition de leur carcasse ont été identiques quel que soit leur âge. L'état d'engraissement initial des animaux étant très comparable pour les 3 premières classes d'âge, cela sous entend donc que les vaches les plus jeunes, dont le gain de poids total en finition a été le plus élevé, ont synthétisé plus de muscles au cours de cette période que leurs homologues âgées de 9 et 10 ans et surtout que celles âgées de 11 à 13 ans. Cette

\begin{tabular}{|l|c|c|c|c|}
\hline Age des animaux & 5 à 8 ans & 9 à 10 ans & 11 à 13 ans & 14 à 16 ans \\
\hline Nombre d'animaux & 11 & 8 & 20 & 10 \\
Etat d'engraissement initial & & & & \\
- note sur 5 & 1,8 & 1,7 & 1,7 & 1,5 \\
- diamètre des adipocytes $(\mu)$ & 51 & 50 & 48 & 47 \\
\hline & & & & \\
Quantités ingérées & & & & \\
- kg MS/j & 10,6 & 10,5 & 10,2 & 9,0 \\
- kg MS/kg de gain & 7,5 & 8,9 & 10,6 & 18,2 \\
Résultats d'engraissement & & & & \\
- poids initial (kg) & 522 & 532 & 549 & 534 \\
- poids final (kg) & 610 & 610 & 610 & 569 \\
- durée (j) & 67 & 66 & 65 & 71 \\
- gain de poids vif (g/j) & 1330 & 1180 & 960 & 495 \\
& & & & \\
\hline Résultats d'abattage & & & & \\
- poids vif vide (kg) & 548 & 544 & 544 & 507 \\
& \pm 38 & \pm 37 & \pm 41 & \pm 28 \\
- poids de carcasse froide $(\mathrm{kg})$ & 345 & 347 & 349 & 330 \\
& \pm 27 & \pm 33 & \pm 29 & \pm 19 \\
- rendement vrai (\%) & 64,0 & 64,5 & 64,5 & 64,9 \\
- dépôts adipeux totaux (kg) ${ }^{(1)}$ & 86 & 89 & 80 & 71 \\
& \pm 28 & \pm 27 & \pm 16 & \pm 14 \\
- dépôts adipeux totaux & 15,5 & 16,2 & 14,6 & 13,9 \\
(en \% du poids vif vide) & $\pm 4,8$ & $\pm 4,7$ & $\pm 2,3$ & $\pm 2,2$ \\
\hline
\end{tabular}

Tableau 8. Influence de l'âge des vaches au moment de leur réforme sur leurs performances d'engraissement. 
observation correspond d'ailleurs parfaitement à l'évolution de l'indice de consommation qui augmente régulièrement (crôtt des animaux plus riche en graisse) avec l'âge des animaux jusqu'à 13 ans. L'indice de consommation très élevé des vaches les plus âgées s'explique par de très fortes variations individuelles, certaines d'entre elles étant incapables de reprendre du poids et de s'engraisser.

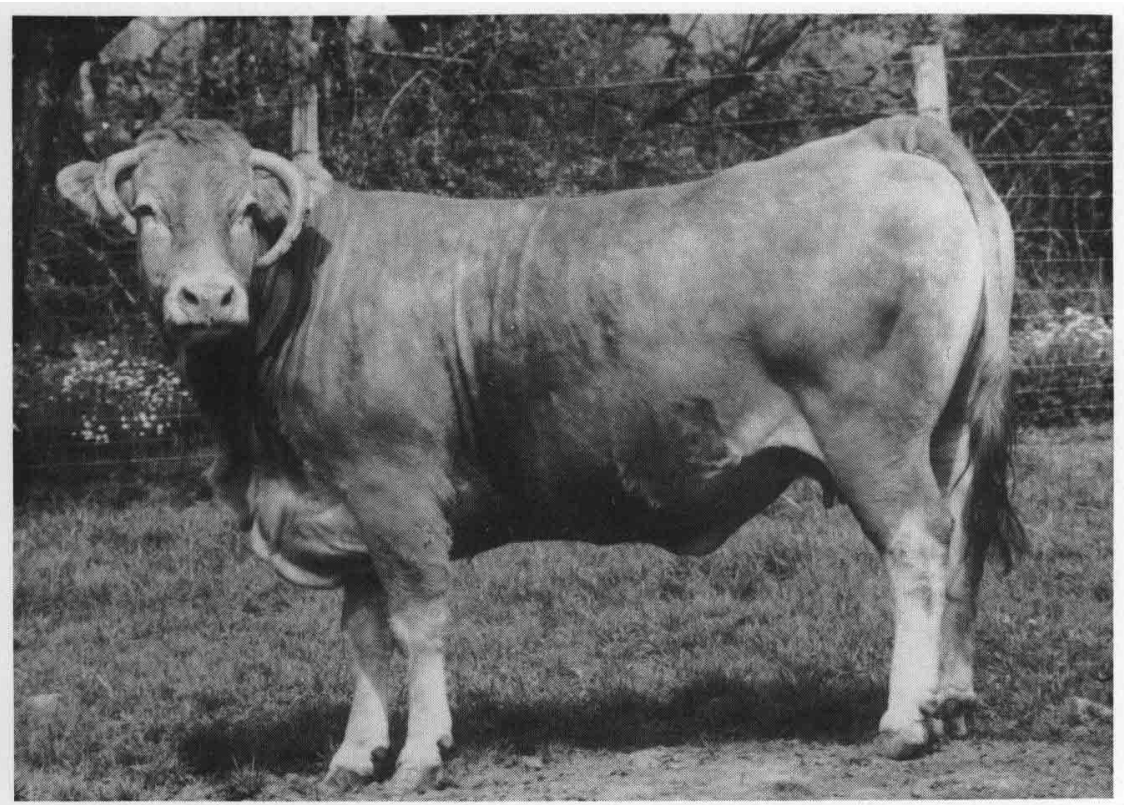

Les performances d'engraissement des animaux très âgés (14 ans et plus) sont médiocres et surtout très variables en fonction du passé de I'animal.

\section{3 / Conclusion}

L'examen de l'ensemble des résultats présentés sur l'engraissement des vaches de réforme de race Limousine montre que des vaches taries et maigres, engraissées à l'auge avec des régimes d'ingestibilité et de concentration énergétique élevées (ensilage de maïs et concentré), peuvent s'engraisser rapidement en 2 mois, 2 mois et demi, et réaliser un gain de poids journalier de l'ordre de $1200 \mathrm{~g}$. Leur gain de carcasse est important, de l'ordre de $50 \mathrm{~kg}$, et la quantité de muscle dans ce gain est voisine de $20 \mathrm{~kg}$. Même si les différentes mesures relatives à la qualité de la viande n'ont pas permis de mettre en évidence de différences entre des vaches maigres et des vaches grasses, l'intérêt de remettre " en état " les vaches de réforme de race Limousine apparait donc clairement. En effet, l'augmentation du poids et l'amélioration de la qualité de carcasse aura des conséquences notables sur le revenu de l'éleveur.

Ces études ont également permis de mesurer avec précision la capacité d'ingestion des vaches Limousines. Comme cela a déjà été observé sur les mâles, les vaches de cette race se caractérisent par une faible capacité d'ingestion (10,5 kg MS soit 1,85 à 1,90 kg MS / 100 $\mathrm{kg}$ de poids vif) qui est inférieure à celle des vaches Charolaises et surtout à celle de vaches Pie-Noires. Leur efficacité alimentaire, en revanche, est de 20 à $30 \%$ supérieure à celle des vaches de réforme de race laitière. Dans ces conditions, il ne paraît pas intéressant de restreindre l'alimentation des animaux au cours de leur période de finition. Leur vitesse de reprise de poids chute en effet considérablement, et il est alors nécessaire de prolonger de façon importante la durée de la période d'engraissement des animaux.

Enfin, une analyse des données individuelles basée sur l'âge des animaux au moment de leur réforme montre que la capacité de reprise de poids des vaches, et donc leur gain de carcasse, sont d'autant plus élevés qu'elles sont jeunes. Toutefois, sur le strict plan de leur finition en boucherie, les vaches de race Limousine sont capables de produire jusqu'à un âge avancé (11-12-13 ans) des carcasses de poids et de composition très satisfaisants. Seules les vaches très âgées, de 14 ans et plus, deviennent difficiles à engraisser et présentent de grandes variations individuelles.

\section{Remerciements}

Cette étude a été commanditée par la Chambre Régionale d'Agriculture du Limousin avec l'aide du FIDAR. Elle a été réalisée en collaboration avec les Chambres d'Agriculture de Corrèze, Creuse et HauteVienne; les lycées agricoles du Limousin ; l'Union régionale des Groupements de Producteurs de Bovins Limousins; l'Institut Technique de l'Elevage Bovin.

\section{Références bibliographiques}

BERANGER C, MALTERRE C., 1968. Influence d'un stéroïde triénique à activité anabolisante sur l'engraissement des vaches taries. Compte rendu des séances de la Société de Biologie. Tome 162, (5-6), 1157-1164.

BERANGER C., NEGRIN M., MALTERRE C., 1970. Evolution du gain de poids vif et de l'état d'engraissement des vaches taries au pâturage. Ann. Zootech., 19 (1), 53-66.

COLLEAU J.J., MALTERRE C., TOURAILLE C., 1984 Influence du type génétique et du niveau de production laitière sur la qualité des carcasses et des viandes de vaches laitières réformées. Bull. Tech. CRZV Theix, INRA, 58, 45-52.

INRA, 1988. Alimentation des bovins, ovins et caprins. $R$ Jarrige éd. INRA Publications, route de St-Cyr, 78026 Versailles Cedex.

KOPP J., BONNET M., 1982. Le tissu conjonctif musculaire. Bull. Tech. CR7V Theix, INRA, 48, 34-37.

LEPETIT J.,1982. Comportement rhéologique du tissu musculaire. Influence des traitements technologiques. Thèse Doct. Ing. Université Clermont-Ferrand 2.

LEPETIT I., CULIOLI J., 1986. Analysis of rheological properties of raw meat. Prospect of application to industrial control of muscles. CEE workshop Accelerated processing. Octobre 1985, Rome.

MALTERRE C., 1972. Peut-on améliorer l'engraissement des vaches laitières réformées. L'Elevage $n^{\circ}$ Hors série "L'exploitation moderne du troupeau laitier », 141-150.

MALTERRE C., 1986. Production de viande de vaches de réforme. in D. Micol éd: "Production de viande bovine », INRA Paris, pp. 247-269.

MALTERRE C., JONES S.D.M., 1990. Meat production from heifers and cull cows, in R. Jarrige éd : « Beef cattle production " Chapter 20. Elsevier à paraître.

ROBELIN J., AGABRIEL J., 1986. Estimation de l'état d'engraissement des bovins vivants à partir de la taille des cellules adipeuses. Bull. Tech. CRZV Theix, INRA, $66,37-41$

ROBELIN J., LESTRADE A., 1982. Variations de la composition corporelle des vaches adultes. Mémoire de DEA Septembre 1982. Université de Clermont-Ferrand.

ROBELIN J., AGABRIEL J., MALTERRE C., BONNE MAIRE J., 1990. Changes in body composition of mature dry cows of Holstein, Limousine and Charolaise breeds during fattening. 1/ Skeleton, muscles, fatty tissues and offal. à paraître. 